\title{
Intravenous Corticotropin-releasing Hormone Administration Increases Esophageal Electrical Sensitivity in Healthy Individuals
}

\author{
Takahisa Yamasaki, Toshihiko Tomita, Mayu Takimoto, Takashi Kondo, Katsuyuki Tozawa, Yoshio Ohda, Tadayuki Oshima, \\ Hirokazu Fukui, Jiro Watari, and Hiroto Miwa* \\ Division of Gastroenterology, Department of Internal Medicine, Hyogo College of Medicine, Nishinomiya, Hyogo, Japan
}

\begin{abstract}
Background/Aims
When a person is experiencing stress, corticotropin-releasing hormone (CRH) can modulate gut physiologies, such as visceral sensation or gastrointestinal motility, and its intravenous administration mimics stress-induced physiological changes. However, the influence of $\mathrm{CRH}$ on the esophagus is yet unknown. Accordingly, we investigated whether intravenous CRH administration increases esophageal sensitivity to electrical stimulation in healthy Japanese subjects.

\section{Methods}

Twenty healthy subjects were recruited. We quantified the initial perception threshold (IPT) every 15 minutes after CRH injection. Venous blood was collected with a cannula, and both plasma adrenocorticotropic hormone (ACTH) and cortisol were measured at pre-stimulation, 0, 30, 60, 90, and 120 minutes. The results from each time point were compared against a baseline IPT obtained before electrical stimulation was initiated.

\section{Results}

When compared to the baseline IPT value (16.9 \pm 4.5$)$, CRH significantly decreased electrical threshold of the esophagus at 30, 45, 60,75 minutes $(14.1 \pm 4.2,13.1 \pm 5.0,12.1 \pm 5.7,14.0 \pm 5.8$ minutes, $P<0.01$, respectively) after $C R H$ injection, suggesting that $\mathrm{CRH}$ increased esophageal sensitivity to the electrical stimulus. CRH also significantly increased plasma ACTH levels at 30 minutes $(50.3 \pm 17.7, P<0.01)$, and cortisol levels at 30 minutes $(22.0 \pm 6.7$ minutes, $P<0.01)$ and 60 minutes $(20.3 \pm 6.7$ minutes, $P<$ 0.01 ) after $\mathrm{CRH}$ injection, when compared to the pre-stimulation ACTH and cortisol values.
\end{abstract}

\section{Conclusion}

Intravenous CRH administration increased esophageal electrical sensitivity in normal subjects, emphasizing the important role of stress in esophageal sensitivity.

(J Neurogastroenterol Motil 2017;23:526-532)

Key Words

Corticotropin-releasing hormone; Electric stimulation; Esophagus

Received: May 25, 2017 Revised: July 12, 2017 Accepted: August 16, 2017

(5) This is an Open Access article distributed under the terms of the Creative Commons Attribution Non-Commercial License (http://creativecommons. org/licenses/by-nc/4.0) which permits unrestricted non-commercial use, distribution, and reproduction in any medium, provided the original work is properly cited.

${ }^{*}$ Correspondence: Hiroto Miwa, MD, PhD

Division of Gastroenterology, Department of Internal Medicine, Hyogo College of Medicine, 1-1, Mukogawa-cho, Nishinomiya, Hyogo 663-8501, Japan

Tel: +81-798-45-6665, Fax: +81-798-45-6661, E-mail: miwahgi@hyo-med.ac.jp

Takahisa Yamasaki and Toshihiko Tomita contributed equally to this work. 


\section{Introduction}

In recent years, as knowledge of functional gastrointestinal disorders (FGID) has advanced, esophageal hypersensitivity has been shown to play a significant role in the onset of upper gastrointestinal tract symptoms, such as heartburn. ${ }^{1}$ However, hypersensitivity is not necessarily common to all nociceptive stimulation; for example, a patient may experience hypersensitivity to mechanical stimuli but not to chemical stimuli. ${ }^{2}$ The pathogenesis of visceral hypersensitivity is mediated by several factors, including peripheral hypersensitivity, central hypersensitivity, and hypersensitivity mediated by psychosocial factors. Peripheral hypersensitivity arises from peripheral sites, such as the esophageal mucosa, and central hypersensitivity occurs in the neurons of the spinal cord and the brain. Psychosocial factors are known to modulate both peripheral and central hypersensitivity. Stress modulates visceral sensation as well as other physiological functions. ${ }^{3}$ The bidirectional association between stress and the gastrointestinal tract has received attention, as researchers have sought to understand how psychological stress can affect the gastrointestinal tract as well as how changes in the gastrointestinal tract can affect stress sensitivity in the brain. ${ }^{4}$ This general interaction among stress, the brain, and the gastrointestinal tract is termed brain-gut interaction. ${ }^{5}$ Corticotropin releasing hormone (CRH), a neuropeptide, is known to be one of the most important factors precipitating the onset of symptoms in stressrelated disease or FGID ${ }^{6,7}$ Gastrointestinal physiologies, such as colonic motor and sensory functions, are known to be altered by stress induced by intravenous administration of $\mathrm{CRH}$ in patients with irritable bowel syndrome (IBS). ${ }^{8,9}$ This evidence suggests that altered brain-gut interactions resulting from a magnified response to $\mathrm{CRH}$ lead to changes in colonic functions and could be relevant to the pathophysiology of IBS. However, the influence of CRH on the esophagus has not yet been fully elucidated. The esophagus has a number of advantages as a site for study of visceral sensation. For example, it is relatively easy to determine the location where esophageal symptoms originate, and a typical symptom is heartburn. Therefore, we aim to further explore this relationship between stress and esophageal function by determining whether intravenous $\mathrm{CRH}$ administration increases esophageal sensitivity to electrical stimulation in healthy Japanese subjects.

\section{Materials and Methods}

\section{Study Design}

This observational study was held in an academic medical center. A total of 20 Japanese volunteers were recruited through advertisements (local newspaper annoucements and in-house leaflets) between October 2012 and April 2013. The frequency scale for the symptoms of gastroesophageal reflux disease (GERD) (FSSG) was validated, ${ }^{10}$ and only subjects with FSSG scores of $0-2$ were recruited. We asked the subjects to fast for at least 6 hours before the examination. An electric catheter was inserted via the nasal passage and was placed in the lower esophagus. Placement was confirmed with an esophageal manometric catheter using high-resolution manometry. We quantified the initial perception threshold (IPT: the minimum current intensity at which the subject first perceived the stimulus). We infused CRH without the subject's knowledge and evaluated IPT. In addition, plasma adreno-corticotropic hormone $(\mathrm{ACTH})$ and cortisol levels were measured using radioimmunoassay pre-stimulation from the start of electrical stimulation until 120 minutes later at 30-minute intervals. These values were compared against a value obtained from a sample taken before the electrical stimulation began. For all subjects, this process was initiated at the same time in the afternoon. This study was approved by the ethics committee of Hyogo College of Medicine (Approval No. 1324), and written informed consent was obtained from all participants.

\section{Healthy Participants}

As noted above, participants were recruited by advertisements. Candidates were excluded from participation if they were younger than 20 years or older than 80 years of age, had overt symptoms of FGID, had used an histamine-2 receptor antagonist or proton pump inhibitors within 2 weeks of the study start date, were regular users of corticosteroids or nonsteroidal anti-inflammatory drugs (NSAIDs) and had used those drugs within the past month, had a history of allergy to drugs, had a history of gastric surgery, had a serious concomitant disease that would affect their ability to complete the study, or were otherwise judged by a physician to be unable to participate in this study.

\section{Electrical Stimulation Test}

To evaluate electrical sensitivity, a 7-Fr electrode (Esogold 4; Taisho Biomed Instruments, Osaka, Japan) was inserted and placed $5 \mathrm{~cm}$ above the superior border of the lower esophageal sphincter. 
Placement was determined using high-resolution manometry (INSIGHTTM Sandhill Scientific, Highlands Ranch, CO, USA). The electrode was connected to an electrical stimulator (SEN-3401; Nihon Kohden, Tokyo, Japan). The waveform was 200 microseconds at a stimulation frequency of $1 \mathrm{~Hz}$. The current intensity was gradually increased by $2 \mathrm{~mA}$ every 20 seconds. Subjects sat upright in a comfortable chair throughout the study. Additionally, electrocardiogram monitoring was performed during the examination. For the end point IPT, we quantified the IPT; that is, the minimum current intensity at which subjects perceived stimulation for the first time by electrical stimulation as it gradually increased. We measured IPT at 15, 30, 45, 60, 75, 90, 105, and 120 minutes after $\mathrm{CRH}$ (Tanabe Mitsubishi Corporation, Osaka, Japan) injection (2 $\mu \mathrm{g}$ / $\mathrm{kg}$ ). We secured vascular access with a saline port to the forearm of the subject at the time of admission. In this way, we could intravenously administer CRH without the subject's knowledge. We prepared CRH 1 hour before administration and preserved it in a refrigerator. IPT was measured 6 times before $\mathrm{CRH}$ administration (every 5 minutes). Baseline IPT was defined as the average of each subject's pre-CRH IPT measurements, excluding the subject's maximum and minimum values. We compared the baseline IPT with the IPT at each time point. Venous blood $(5 \mathrm{~mL})$ was obtained via a cannula placed in the antebrachial vein, and plasma concentrations of ACTH and cortisol were measured by radioimmunoassay at pre-stimulation, 0, 30, 60, 90, and 120 minutes after CRH injection (Fig. 1). We compared the pre-stimulation value with those values.

\section{Outcome Measures}

The primary end point was the esophageal IPT after electrical stimulation. The secondary end points were the plasma ACTH and cortisol levels after electrical stimulation, which were compared against pre-stimulation values.

\section{Statistical Methods}

All results are expressed as the mean \pm standard deviation. Statistical differences in IPT between participants were determined using a two-way analysis of variance (ANOVA). Statistical significance was defined as a value of $P<0.05$.

\section{Results}

\section{Enrollment and Baseline Characteristics of the Participants}

Twenty subjects were recruited to this study. There were 19 men and 1 woman, with a mean age of $35.1 \pm 7.5$ (range, 27-51) years. The mean body mass index of all subjects was $23.1 \pm 1.8 \mathrm{~kg} /$ $\mathrm{m}^{2}$. There was a history of drinking alcohol in 7 subjects, and a history of smoking in 5 subjects (Table).

\section{Corticotropin-releasing Hormone Increased Esophageal Sensitivity to the Electrical Stimulus}

The words most commonly used by subjects to describe the sensation elicited by electrical stimulation of the esophagus were "pulsing," "sharp," and "throbbing." When compared to baseline IPT $(16.9 \pm 4.5), \mathrm{CRH}$ significantly decreased the electrical

Table. Characteristics of Subjects

\begin{tabular}{lc}
\hline & Total $(\mathrm{N}=20)$ \\
\hline Gender $(\mathrm{M}: \mathrm{F})$ & $19: 1$ \\
BMI $\left(\mathrm{kg} / \mathrm{m}^{2}\right)$ & $23.1 \pm 1.8$ \\
Drinking habit & 7 \\
Smoking habit & 5 \\
\hline
\end{tabular}

BMI, body mass index.

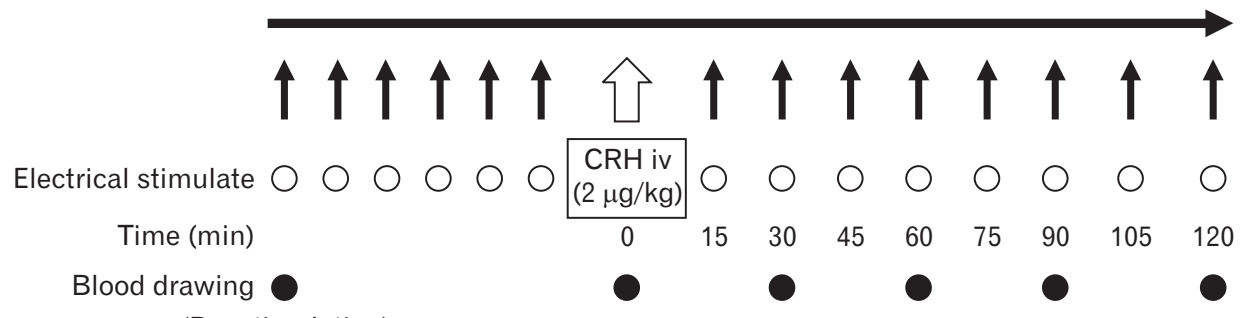

(Pre-stimulation)

Figure 1. Study protocol. We measured the the initial perception threshold (IPT) at 15, 30, 45, 60, 75, 90, 105, 120 minutes after corticotropinreleasing hormone $(\mathrm{CRH})$ injection. IPT was measured 6 times before CRH injection. Then, baseline IPT was defined as the average of IPTs except for maximum and minimum IPTs. Blood was drawn and plasma adrenocorticotropic hormone and cortisol were measured at 0, 30, 60, 90, and 120 minutes after CRH injection. iv, intravenous. 
stimulation threshold of the esophagus at 30 minutes $(14.1 \pm 4.2$, $P<0.01), 45$ minutes $(13.1 \pm 5.0, P<0.01), 60$ minutes $(12.1$ \pm 5.7, $P<0.01)$, and 75 minutes $(14.0 \pm 5.8, P<0.01)$ after $\mathrm{CRH}$ injection. IPT was lowest at 60 minutes after $\mathrm{CRH}$ injection. Thereafter, it gradually recovered showing a similar value to the baseline IPT at 120 minutes $(16.5 \pm 4.7)$ after CRH injection (Fig. 2).

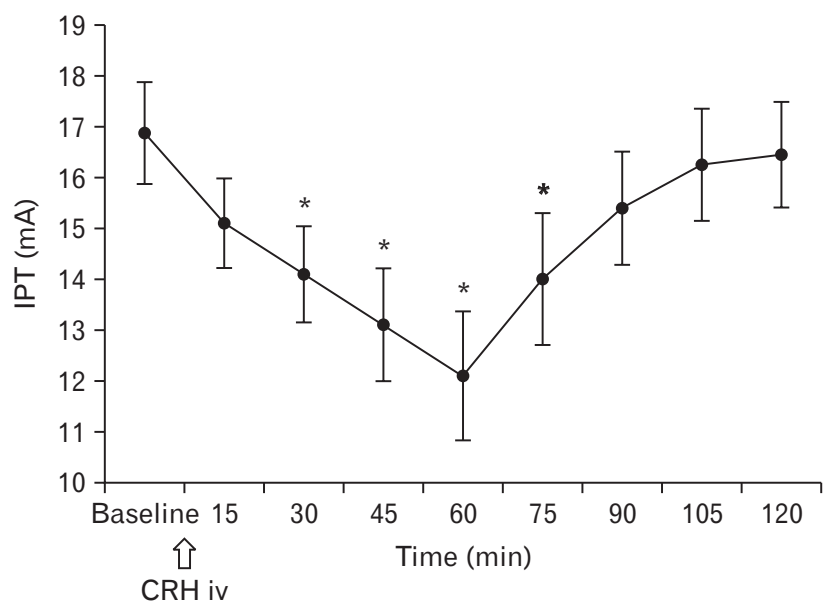

Figure 2. Changes in esophageal sensitivity to the electrical stimulus induced by corticotropin-releasing hormone (CRH). CRH significantly decreased electrical threshold of the esophagus after $\mathrm{CRH}$ injection. IPT, initial perception threshold; iv, intravenous. ${ }^{*} P<0.01$.

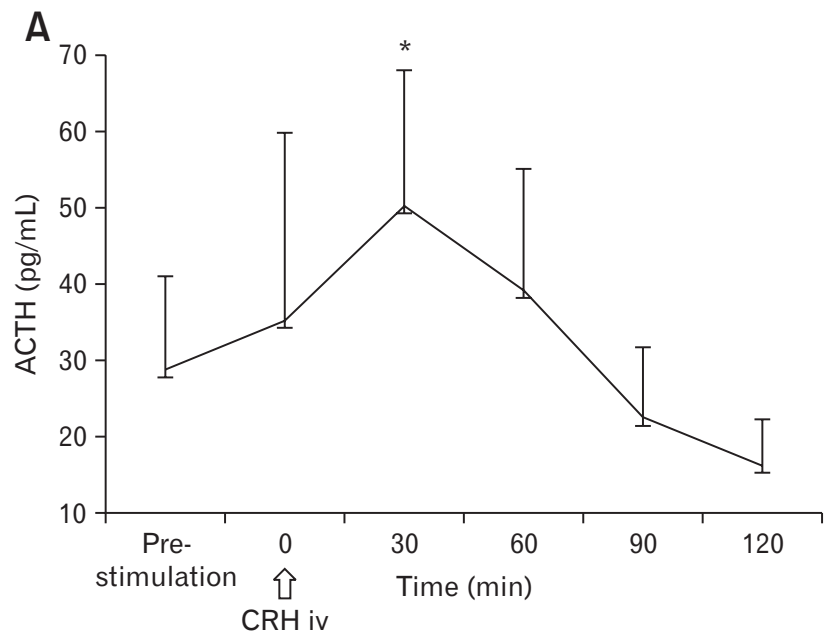

\section{Corticotropin-releasing Hormone Significantly Increased Plasma Adreno-corticotropic Hormone and Plasma Cortisol Levels Compared to Pre-stimulation Levels}

$\mathrm{CRH}$ significantly increased plasma ACTH levels at 30 minutes $(50.3 \pm 17.7, P<0.01)$ after $\mathrm{CRH}$ injection, compared to pre-stimulation ACTH values $(28.7 \pm 12.3)$ (Fig. 3A). Additionally, cortisol levels at 30 minutes $(22.0 \pm 6.7, P<0.01)$ and 60 minutes $(20.3 \pm 6.7, P<0.01)$ after $\mathrm{CRH}$ injection were significantly increased compared to the pre-stimulation cortisol values $(13.8 \pm 4.6)$ (Fig. 3B). There was no correlation between degree of ACTH/cortisol change and degree of IPT change.

\section{Discussion}

This is the first reported study in which a simulated stress load was caused by intravenous administration of $\mathrm{CRH}$, which is considered physiologically equivalent to stress, to analyze esophageal perception in healthy Japanese individuals by using an electrical stimulation test. A recent study from Belgium demonstrated that intravenous $\mathrm{CRH}$ administration increased esophageal sensitivity to mechanical distention in healthy subjects. ${ }^{11}$ Our study demonstrated that acute stress, simulated by intravenous CRH, significantly decreased the electrical threshold of esophageal perception. In addition, to the IPT, we also measured ACTH and cortisol levels to verify that subjects were exposed to a true stress load.

Recent studies have reported that an important factor in the

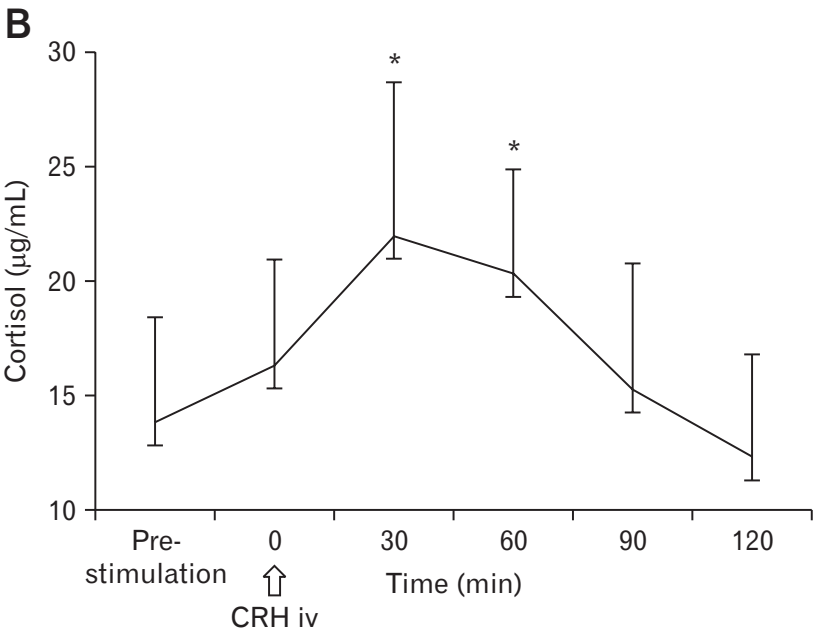

Figure 3. Effects of corticotropin-releasing hormone (CRH) on plasma ACTH and plasma cortisol levels. (A) CRH significantly increased plasma ACTH levels 30 minutes after CRH injection, when compared to pre-stimulation ACTH value. (B) Plasma cortisol levels 30 minutes and 60 minutes after $\mathrm{CRH}$ injection were significantly increased compared to pre-stimulation cortisol values. iv, intravenous. ${ }^{*} P<0.01$. 
pathogenesis of non-erosive reflux disease (NERD), functional dyspepsia, and IBS may be hyperresponsiveness to stress in the gastrointestinal tract. ${ }^{912-14}$ In daily life, we experience various symptoms due to excessive "stress" on the body. When the human brain perceives stress, $\mathrm{CRH}$ is released from the hypothalamus. $\mathrm{CRH}$ acts on the pituitary gland to secrete $\mathrm{ACTH}$, which induces the secretion of cortisol from the adrenal cortex, resulting in various physiological reactions necessary for adaptation to stress. ${ }^{6}$ Central and gastrointestinal responses to stress can be pronounced, particularly in patients with FGID such as IBS. $7,8,15,16$

The association between stress and visceral perception has been reported in a number of colonic studies. Fukudo et $\mathrm{al}^{17}$ showed that peripherally administered $\mathrm{CRH}$ induced more frequent bowel movements in patients with IBS as compared to healthy individuals. One study demonstrated a greater increase in perception of colonic distension after intravenous $\mathrm{CRH}$ administration in healthy individuals, in contrast to the response prior to administration or following placebo. ${ }^{18}$ Another study reported that when $\alpha$-helical CRH was peripherally administered in IBS patients, the hyperresponsiveness to stress induced by colonic electrical stimulation was suppressed. ${ }^{8}$ These results indicate that stress is closely associated with colonic perception and bowel movements.

Previous animal studies have analyzed esophageal perception after stress loading. Stress induced by $\mathrm{CRH}$ administration in rats causes acute enlargement of the esophageal intercellular space, which may be associated with the pathogenesis of GERD. ${ }^{19}$ It has also been reported that $\mathrm{CRH}$ affects gastric peristalsis in rats. ${ }^{20-22}$ No study on the effect of intraesophageal stimulation following stress loading with $\mathrm{CRH}$ has been reported in humans. In the present study, we analyzed the threshold of esophageal perception and measured ACTH and cortisol levels in response to electrical stimulation, before and after acute stress loading with intravenous CRH. The aim was to determine whether acute stress enhances esophageal perception.

Several studies have characterized aspects of visceral hyperesthesia in esophageal disease. A study of mechanosensitivity using an esophageal barostat showed that NERD patients felt pain in response to a significantly smaller volume of instilled air than healthy individuals, ${ }^{23}$ indicating that NERD patients are significantly more sensitive to mechanical stimulation. Using an esophageal acid perfusion test in Japanese, we observed a significantly shorter time to pain onset and greater pain due to acid stimulation in patients with NERD as compared to patients with erosive esophagitis or healthy individuals. In addition, sensory scores, which are the mathematical products of pain onset and pain levels, were significantly higher than healthy individuals. ${ }^{24}$ These results indicate that sensitivity to intraesophageal acid stimulation is clearly higher in NERD patients than healthy individuals.

In the present study, we used a previously employed electrical method that directly stimulates afferent nerves rather than receptors, ${ }^{25,26}$ to analyze esophageal perception. Prior methods used to evaluate the threshold of esophageal perception have included chemical stimulation, an acid perfusion test, a mechanical stimulation method, and a barostat test. Each method has advantages and disadvantages with regard to simplicity, reproducibility, and invasiveness. ${ }^{27}$ Catheters used in electrical stimulation tests can be easily inserted, as they have significantly smaller diameters than barostat catheters. Thus, the degree of invasiveness is relatively low with the electrical stimulation method. Moreover, whether subjects actually perceive esophageal stimulation can be verified by simply adjusting the intensity of the stimulation current yielding a high level of reproducibility.

It is important to analyze the kinetics of $\mathrm{ACTH}$ after $\mathrm{CRH}$ administration, as an increase in $\mathrm{ACTH}$ confirms exposure to stress. ${ }^{28}$ In the present study, we were able to analyze ACTH kinetics using intravenous $\mathrm{CRH}$ after electrical stimulation, enabling us to show that subjects underwent the electrical stimulation test under conditions of stress. Therefore, we consider the results of the present study to be reliable. CRH administration may be useful in identifying the onset of stress and the threshold of esophageal perception in a clinical setting. This method suggests the possibility of investigating the difference in the response to stress between individuals as well as between organs.

There are several limitations in the present study. First, this was an observational study performed at a single institution. In addition, we performed analyses in healthy individuals rather than patients. Therefore, it remains unclear whether the values for the IPT caused by $\mathrm{CRH}$ administration in healthy individuals are applicable to hypersensitive patients, such as those with NERD, and whether the IPT values in patients are similar to those in healthy individuals, or instead indicate greater sensitivity than in healthy individuals. This is a subject for future study.

The small number of subjects $(\mathrm{n}=20)$ may have affected the results. Furthermore, the majority of enrolled subjects in the present study were men. These are considered limitations, since gender differences in $\mathrm{CRH}$ reactivity have been demonstrated. ${ }^{29,30}$ In these studies, women were shown to be more sensitive to stimulation than men. In the present study, 19 of 20 subjects were men.

In conclusion, our study demonstrated that acute stress significantly decreases the threshold of esophageal electrical sensitivity in 
healthy subjects, indicating that $\mathrm{CRH}$ plays an important role in the brain-gut interaction. The effect of $\mathrm{CRH}$ in both healthy individuals and patients with FGID, as well as in those with GERD, will be further explained by future studies.

\section{Financial support: None.}

\section{Conflicts of interest: None.}

Author contributions: Takahisa Yamasaki and Toshihiko Tomita contributed to the concept and design of the study; Takahisa Yamasaki, Toshihiko Tomita, and Mayu Takimoto were involved in data acquisition and analysis; Tadayuki Oshima provided statistifical design; Hiroto Miwa coordinated acquisition of data and trial supervision; and all authors contributed to data interpretation, critically reviewed the manuscript for important intellectual contents, and approved final version of the manuscript.

\section{References}

1. Miwa H, Kondo T, Oshima T, Fukui H, Tomita T, Watari J. Esophageal sensation and esophageal hypersensitivity - overview from bench to bedside. J Neurogastroenterol Motil 2010;16:353-362.

2. Fass R, Naliboff B, Higa L, et al. Differential effect of long-term esophageal acid exposure on mechanosensitivity and chemosensitivity in humans. Gastroenterology 1998;115:1363-1373.

3. Mertz H. Review article: visceral hypersensitivity. Aliment Pharmacol Ther 2003;17:623-633.

4. Bhatia V, Tandon RK. Stress and the gastrointestinal tract. J Gastroenterol Hepatol 2005;20:332-339.

5. Aziz Q, Thompson DG. Brain-gut axis in health and disease. Gastroenterology 1998;114:559-578.

6. Heinrichs SC, Menzaghi F, Merlo Pich E, Britton KT, Koob GF. The role of CRF in behavioral aspects of stress. Ann N Y Acad Sci 1995;771:92-104.

7. Nozu T, Okumura T. Corticotropin-releasing factor receptor type 1 and type 2 interaction in irritable bowel syndrome. J Gastroenterol 2015;50:819-830.

8. Sagami Y, Shimada Y, Tayama J, et al. Effect of a corticotropin releasing hormone receptor antagonist on colonic sensory and motor function in patients with irritable bowel syndrome. Gut 2004;53:958-964.

9. Taché Y, Million M. Role of corticotropin-releasing factor signaling in stress-related alterations of colonic motility and hyperalgesia. J Neurogastroenterol Motil 2015;21:8-24.

10. Kusano M, Shimoyama Y, Sugimoto S, et al. Development and evaluation of FSSG: frequency scale for the symptoms of GERD. J Gastroenterol 2004;39:888-891.

11. Broers C, Melchior C, Van Oudenhove L, et al. The effect of intravenous corticotropin-releasing hormone administration on esophageal sensitivity and motility in health. Am J Physiol Gastrointest Liver Physiol 2017;312:G526-G534.

12. Mayer EA. The neurobiology of stress and gastrointestinal disease. Gut 2000;47:861-869.

13. Geeraerts B, Vandenberghe J, Van Oudenhove L, et al. Influence of experimentally induced anxiety on gastric sensorimotor function in humans. Gastroenterology 2005;129:1437-1444.

14. Van Oudenhove L, Vandenberghe J, Geeraerts B, et al. Determinants of symptoms in functional dyspepsia: gastric sensorimotor function, psychosocial factors or somatisation? Gut 2008;57:1666-1673.

15. Kanazawa M, Hongo M, Fukudo S. Visceral hypersensitivity in irritable bowel syndrome. J Gastroenterol Hepatol 2011;26:119-121.

16. Lee YJ, Park KS. Irritable bowel syndrome: emerging paradigm in pathophysiology. World J Gastroenterol 2014;20:2456-2469.

17. Fukudo S, Nomura T, Hongo M. Impact of corticotropin-releasing hormone on gastrointestinal motility and adrenocorticotropic hormone in normal controls and patients with irritable bowel syndrome. Gut 1998;42:845-849.

18. Nozu T, Kudaira M. Corticotropin-releasing factor induces rectal hypersensitivity after repetitive painful rectal distention in healthy humans. J Gastroenterol 2006;41:740-744.

19. Cho YJ, Kim JH, Yim HE, Lee DM, Im SK, Lee KJ. Role of corticotrophin-releasing factor in the stress-induced dilation of esophageal intercellular spaces. J Korean Med Sci 2011;26:279-283.

20. Martínez V, Rivier J, Wang L, Taché Y. Central injection of a new corticotropin-releasing factor (CRF) antagonist, astressin, blocks CRF- and stress-related alterations of gastric and colonic motor function. J Pharmacol Exp Ther 1997;280:754-760.

21. Hirata T, Keto Y, Yamano M, Yokoyama T, Sengoku T, Seki N. Inhibitory effect of ramosetron on corticotropin releasing factor- and soybean oil-induced delays in gastric emptying in rats. J Gastroenterol Hepatol 2012;27:1505-1511.

22. Nozu T, Tsuchiya Y, Kumei S, Takakusaki K, Okumura T. Peripheral corticotropin-releasing factor (CRF) induces stimulation of gastric contractions in freely moving conscious rats: role of CRF receptor types 1 and 2. Neurogastroenterol Motil 2013;25:190-197.

23. Trimble KC, Pryde A, Heading RC. Lowered oesophageal sensory thresholds in patients with symptomatic but not excess gastro-oesophageal reflux: evidence for a spectrum of visceral sensitivity in GORD. Gut 1995;37:7-12.

24. Miwa H, Minoo T, Hojo M, et al. Oesophageal hypersensitivity in Japanese patients with non-erosive gastro-oesophageal reflux diseases. Aliment Pharmacol Ther 2004;20(suppl 1):112-117.

25. Sarkar S, Aziz Q, Woolf CJ, Hobson AR, Thompson DG. Contribution of central sensitisation to the development of non-cardiac chest pain. Lancet 2000;356:1154-1159.

26. Anand P, Aziz Q, Willert R, van Oudenhove L. Peripheral and central mechanisms of visceral sensitization in man. Neurogastroenterol Motil 2007;19(1 suppl):29-46.

27. Fass R. Sensory testing of the esophagus. J Clin Gastroenterol 2004;38:628-641.

28. Abelson JL, Khan S, Young EA, Liberzon I. Cognitive modulation of 
endocrine responses to $\mathrm{CRH}$ stimulation in healthy subjects. Psychoneuroendocrinology 2010;35:451-459.

29. Buffalari DM, Baldwin CK, Feltenstein MW, See RE. Corticotrophin releasing factor $(\mathrm{CRF})$ induced reinstatement of cocaine seeking in male and female rats. Physiol Behav 2012;105:209-214.
30. McRae-Clark AL, Cason AM, Kohtz AS, Moran Santa-Maria M, Aston-Jones G, Brady KT. Impact of gender on corticotropin-releasing factor and noradrenergic sensitivity in cocaine use disorder. J Neurosci Res 2017;95:320-327. 